\title{
Modelling and Analysis of a Plus-Shaped PN Junction Phase Shifter for Data Centre Applications
}

\author{
R.G. JESUWANTH SUGESH ${ }^{1 *}$, A. Sivasubramanian ${ }^{2}$ \\ ${ }^{1 *}$ Research Scholar, Vellore Institute of Technology, School of Electronics Engineering, Chennai, \\ INDIA \\ ${ }^{2}$ Vellore Institute of Technology, School of Electronics Engineering, Chennai, INDIA
}

\begin{abstract}
Scaling up of photonic devices is the current research of interest to meet the alarming demand growth in the data centres. The efficiency of the modulator is determined by the performance of the phase shifter. In this paper, a plus-shaped PN junction phase shifter is designed and analysed. This design improved the modulation efficiency and reduced optical loss for high-speed data operation. The width of the $\mathrm{P}$ doped region and thickness of thedoped regions in the slabs are varied to obtain high modulation efficiency. The circuit-level simulation analysis was performed on the proposed phase shifterimported in a travelling wave electrode silicon Mach Zehnder modulator. At 80 Gbps, a maximum extinction ratio of $12.39 \mathrm{~dB}$ with a bit error rate of $8.67 \times 10-8$ was obtained at $\mathrm{V} \pi \mathrm{L} \pi$ of 1.05 V.cm for the length of the phase shifter of $3.5 \mathrm{~mm}$. The calculated intrinsic $3 \mathrm{~dB}$ bandwidth is $\sim 38$ $\mathrm{GHz}$ and the energy per bit transmission is $1.71 \mathrm{pJ} /$ bit.Further analysis was performed to identify the maximum communication distance supported by this proposed phase shifter design in the silicon Mach Zehnder modulator for the data centre requirements.
\end{abstract}

Keywords - PN junction phase shifter, Silicon Mach- Zehnder modulator, optical communication system, silicon photonic device, and data centre application.

Received: December 2, 2020. Revised: April 22, 2021. Accepted: May 13, 2021. Published: May 31, 2021.

\section{Introduction}

Technological advancements such as Artificial Intelligence, Internet of Things, 5G, etc. have paved the way for the increase in internet traffic. Internet traffic is growing at an alarming rate due to the increase in file sharing, video calls, online conferences, meetings, video streaming, and business transactions and so on [1]. This increase in network traffic has put pressure on the data centres that process these requests. International Telecommunication Union has provided regulations in utilising the optical bandwidth. To meet the futuristic network demand, Silicon photonics $(\mathrm{SiPh})$ technology has been introduced. $\mathrm{Si} \mathrm{Ph}$ provides a costeffective method to integrate the electronic and photonic components on a Si chip utilising the advantages of the CMOS fabrication technology [2] \& [3].

For high-speed data transmission link between electronic and optic components, the optical modulator plays a vital role [4 -6]. Hybrid optical modulators with materials such as Indium Tin Oxide, graphene, [7 - 13] etc. provide high modulation efficiency but are not CMOS fabrication compatible as silicon. Modulation in silicon optical modulators follows the principle of plasma dispersion effect. The external voltage bias causes a carrier concentration change in the doped region which in turns leads to the effective index variation [14]. Carrier injection by forward bias voltage and carrier depletion by reverse bias voltage is the standard plasma dispersion techniques used. The carrier injection technique has a drawback of low $3 \mathrm{~dB}$ bandwidth due to high diffusion junction capacitance and long free-carrier lifetime, even though high modulation efficiency (low $\mathrm{V} \pi \mathrm{L}$ ) is obtained [4]. The carrier depletion techniques (CDT) overcomes this drawback and also supports high-speed data transmission [15] \& [16]. The disadvantage in carrier depletion is the low modulation efficiency (high $\mathrm{V} \pi \mathrm{L}$ ). Among the optical modulators available Mach Zehnder modulator (MZM) is preferred for its thermal stability, ease of fabrication and high performance. The shortcoming in an MZM is the high power consumption and large footprint [17].

The modulator's performance is based on the efficiency of the phase shifter, which is based on the doping pattern and concentration used. Based on the doping pattern, the phase shifter is classified into interleaved, vertical and horizontal type [18 - 25]. Multiple PN junctions along the phase shifter length provide high modulation efficiency in interleaved type phase shifter but at the expense of fabrication complexity. Horizontal type phase shifters reduced the fabrication complexity and used low doping concentration at the cost of phase shifter length. The phase shifter length was reduced in vertical type by using high doping concentrated vertical slabs. Free carrier absorption loss increases with the increase of carrier concentration. Slot like structures were used in [2628 ] to reduce the loss and to increase the light-matter 
interaction. Travelling wave electrode (TWE) design is preferred over lumped design, as they are RC time constant independent. TWE supports CDT mode operation [29-31] but has a large footprint.

By reducing the doping region and utilising the advantages of horizontal and vertical type doping in a phase shifter, a novel plus-shaped PN junction phase shifter design with high modulation efficiency is proposed. The parameters that influence the design structure are discussed in section II. The objective is to design an optimised carrier depletion type plusshaped phase shifter with very low $\mathrm{V} \pi \mathrm{L}$, and thus the drawback of the MZM is overcome. Silicon MZM with the proposed phase shifter will meet the demands of the highspeed data transmission in inter and intra data centre applications. The simulation results are analysed in section III.

\section{Device Structure}

A rib waveguide with width $500 \mathrm{~nm}\left(\mathrm{~W}_{\text {rib }}\right)$, ridge thickness $220 \mathrm{~nm}\left(\mathrm{t}_{\text {rib }}\right)$ with etching depth of $90 \mathrm{~nm}$ is considered for the work as it is of fabrication standards and offers better optical confinement with single-mode $\left(\mathrm{TE}_{1}\right)$ operation.

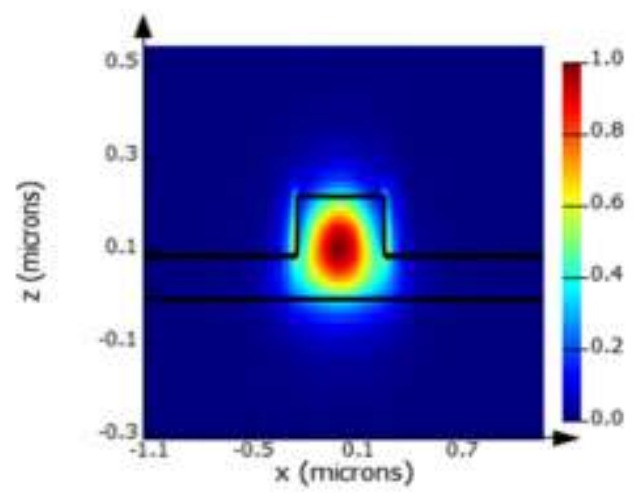

Fig 1: Light propagation through the rib waveguide $(500 \times 220 \mathrm{~nm})$

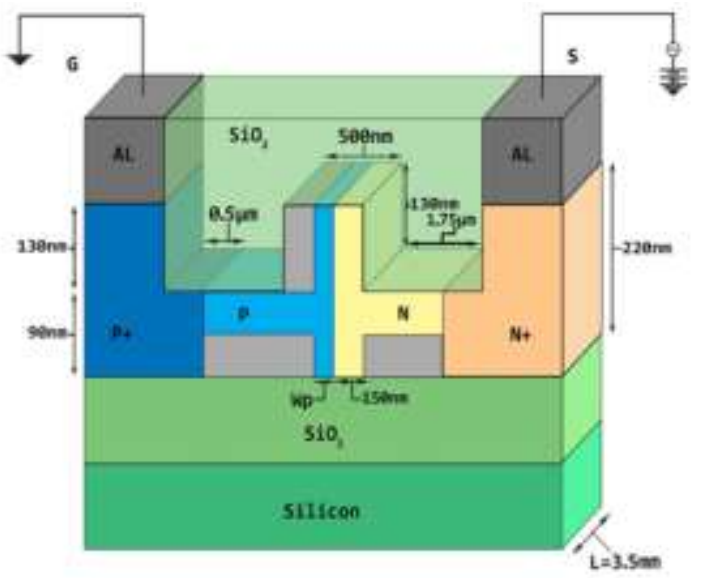

Fig 2: 3D view of the plus-shaped phase shifter

The simulation analysis was performed in an analytical tool [32].The light propagation through the waveguide is shown in figure 1 . An offset of $100 \mathrm{~nm}$ from the centre of the waveguide is followed for the $\mathrm{PN}$ junction to improve the phase efficiency. The width of the $\mathrm{N}$ doped region is set to $150 \mathrm{~nm}$ and the thickness of (P and N) slabs set to 70nm. Since holes have a larger index shift, and lower absorption than electrons, [7] the concentrations of $\mathrm{P}$ and $\mathrm{N}$ dopants are set to $9 \times 10^{17} \mathrm{~cm}$ ${ }^{3}$ and $7 \times 10^{17} \mathrm{~cm}^{-3}$, respectively. Slab resistance is reduced with $1 \times 10^{19} \mathrm{~cm}^{-3}$ carrier concentration of $\mathrm{P}+$ and $\mathrm{N}+$ doped region at the edge of the waveguide. Aluminium electrodes (Al) are used as electrical contacts. The length of the phase shifter (L) was fixed to $3.5 \mathrm{~mm}$ and the $3 \mathrm{D}$ view of the plus-shaped phase shifter is shown in figure 2.To keep power consumption further minimum, the driving voltage of a maximum $5 \mathrm{~V}$ is applied to the cathode ( $\mathrm{V}=0$ to $5 \mathrm{~V})$.

\section{Result Analysis}

The analysis was classified into two parts, device-level simulation and system-level simulation (Fig. 3). In the devicelevel simulation, the electro-optic and the RF characteristics of the proposed phase shifter (PS) was performed using the finite-difference eigen mode (FDE) analysis (Fig 3.a). For system-level analysis, the designed phase shifter parameters were imported into MZM (PS-MZM) in Lumerical Interconnect (Fig 3.b). High-speed data rate and long-distance transmission analysis were also performed to analyse the PSMZM's performance for data centre on and off-chip transmission application respectively.
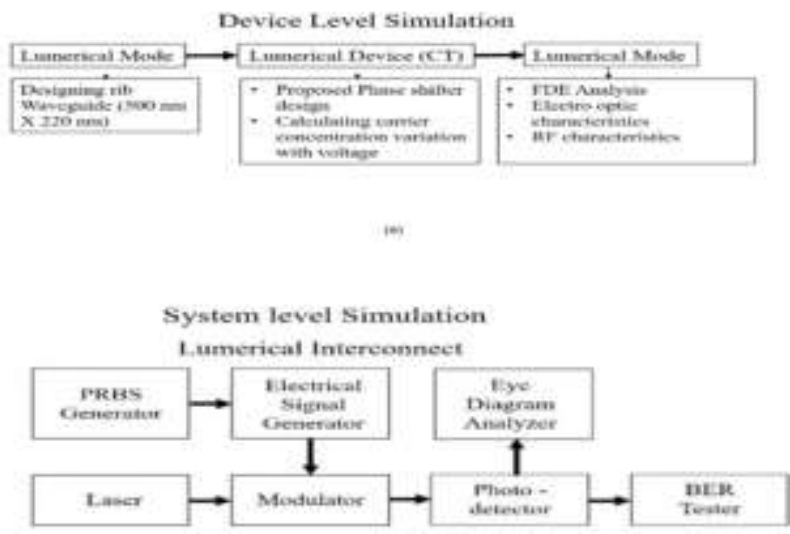

Fig 3: Simulation flow for result analysis (a) Device-level simulation process (b) System-level simulation process

\section{a. Device level analysis}

The reverse bias voltage to the phase shifter was varied to analyse the electro-optic performance of the phase shifter. When the bias voltage is increased, the carriers are depleted from the PN junction. This reduces the carrier density, and thus the capacitance in the junction is reduced as shown in Figure 4. Thus the capacitance of the junction (C) is based on the hole $\left(N_{p}\right)$ and electron $\left(N_{n}\right)$ density $(1)$,

$C=\frac{t_{r i b} \times \sqrt{ }\left(q \varepsilon_{o} \varepsilon_{r}\right)}{\sqrt{\left(2\left(N_{p}^{-1}+N_{n}^{-1}\right) \times\left(V_{v}-V\right)\right)}}$

where $q$ denotes the electric charge, $\varepsilon_{0}$ the dielectric constant, $\varepsilon_{r}$ therelative permittivity and $V_{v}$ the diffusion potential. 


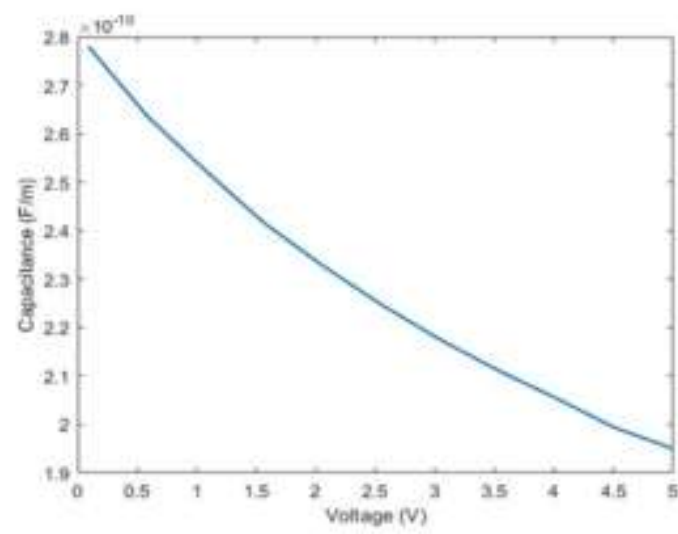

Fig 4: Capacitance of the junction of the proposed phase shifter for varying bias voltage

Carrier depletion has an impact on the absorption coefficient $(\Delta \alpha)$ and refractive index $(\Delta \mathrm{n})$ of the phase shifter. Their mathematical relationship ((2) and (3)) was expressed by Soref and Bennett in [14].

$$
\begin{aligned}
& \Delta \mathrm{n}=-8.8 \times 10^{-22} N_{n}-8.5 \times 10^{-18} N_{p}^{0.8} \\
& \Delta \alpha=8.5 \times 10^{-18} N_{n}+6 \times 10^{-18} N_{p}
\end{aligned}
$$

The optical property of the Phase shifter is altered by the effective index change (5) with voltage $\left(\Delta n_{e f f}(V)\right)$. This causes a phase shift $(\varphi)$ on the propagating optical wave along the phase shifter length $(L)$, which is calculated by (6).

$$
n_{e f f}(V)=n_{e f f, i}+\int \Delta n(V) d V
$$

where $n_{\text {eff }, i}$ denotes the effective index of the waveguide without doping.

$$
\begin{gathered}
\Delta n_{e f f}(V)=n_{e f f}(V)-n_{e f f}(0) \\
\varphi(V)=\frac{2 \pi \Delta n_{e f f}(V)}{\lambda L}
\end{gathered}
$$

The absorption coefficient $\alpha$ along the PS (z-axis) is obtained from (7).

$\alpha(\mathrm{k})=\frac{\iint \Delta \alpha(\mathrm{V})\left|\mathrm{E}(\mathrm{x}, \mathrm{y}, \mathrm{z})^{2}\right| \mathrm{dxdy}}{\iint\left|\mathrm{E}(\mathrm{x}, \mathrm{y}, \mathrm{z})^{2}\right| d x d y}$

where $\mathrm{x}, \mathrm{y}$ denotes the waveguide dimension coordinates, and $\mathrm{z}$ denotes the length coordinate of the phase shifter, and $E(x, y, z)$ the optical intensity distribution of the waveguide mode.

The absorption loss contributes to the significant portion of the total loss in the phase shifter. Absorption loss is due to the absorption of photons by the carriers (free carrier absorption loss). With the increase in voltage, the carriers are depleted from the junction, and this reduces the carrier absorption loss along the phase shifter (Figure 5). When the $\mathrm{P}$ doped region
(Wp) is large, the free carriers present were high thus, the loss is high though the phase shift is obtained at lower voltages. When the $\mathrm{P}$ doped region is small, the loss is reduced, and due to very low carrier concentration variation, the required phase shift obtained is at a higher voltage. It is observed that with $\mathrm{Wp}=100 \mathrm{~nm}$ a lower loss and required $\pi$ phase shift is obtained within the voltage limits set. When reducing the $\mathrm{Wp}$ less than 100nm the loss is reduced but at the cost of phase efficiency. The required phase shift for modulation is obtained at a reverse bias voltage of $3.05 \mathrm{~V}(\mathrm{~V} \pi)$ in the proposed design for $3.5 \mathrm{~mm}$ phase shifter length with a loss of $4.1 \mathrm{~dB}$. The variation of $\mathrm{V} \pi$ along with the loss as a function of the phase shifter length is shown in figure 6. With the increase in length, $\mathrm{V} \pi$ reduces as the propagating optical wave is exposed to the phase shift change throughout the phase shifter length. But as the interaction of the carriers with the optical mode is increased with length, the loss increases with length and operating speed decreases. This forms the trade-off condition between $\mathrm{V} \pi$ and length of the phase shifter.

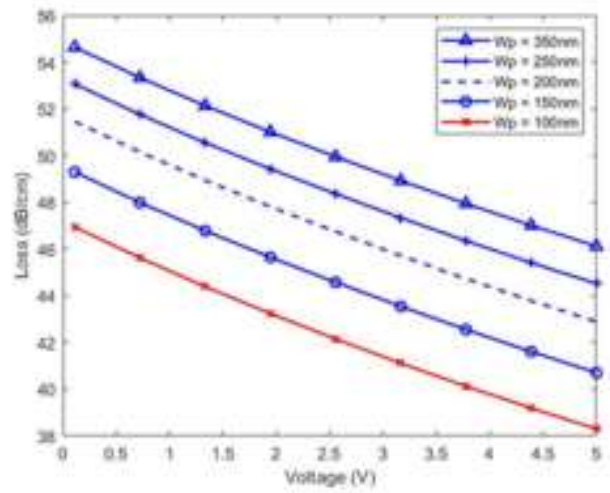

Fig 5: Loss vs voltage for varying $\mathrm{Wp}$ in the proposed phase shifter design of length $3.5 \mathrm{~mm}$

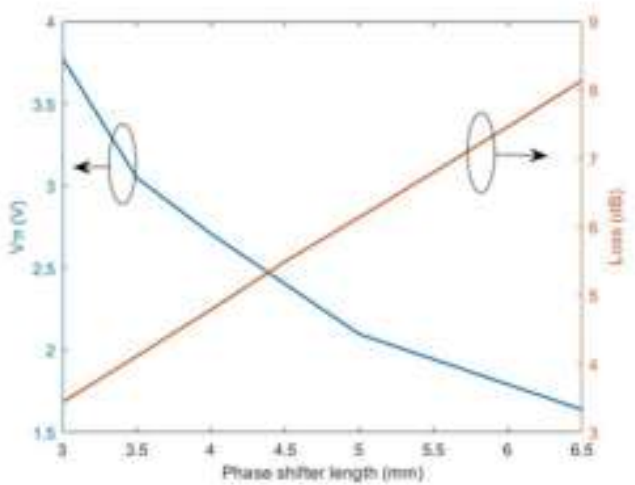

Fig 6: V $\pi$ and loss variation with respect to the length of the phase shifter for the proposed phase shifter design with $\mathrm{Wp}=100 \mathrm{~nm}$

At ideal conditions, the $3 \mathrm{~dB}$ intrinsic bandwidth $\left(f_{3 \mathrm{~dB}}=\right.$ $\left.\frac{1}{2 \pi R C}\right)$ of the proposed phase shifter is calculated to be $37.7 \mathrm{GHz}$. A travelling wave electrode is introduced to overcome RC time constant and to tightly couple the RF microwave with the optical wave. For strong coupling, the optical group index (OGI) has to be equal to the effective index at a particular frequency. At $26 \mathrm{GHz}$, the index matching takes place, leading to the maximum coupling between RF 
mode and optical mode. $3 \mathrm{~dB} / \mathrm{cm}$ loss was calculated at $26 \mathrm{GHz}$, as shown in figure 7 , and it is witnessed that loss is directly proportional to microwave frequency. $6 \mathrm{~dB}$ bandwidth of $24.75 \mathrm{GHz}$ is attained for the designed structure of length $3.5 \mathrm{~mm}$ (Fig. 8) and ensures that the device can be utilised for high-speed data rate communication applications.

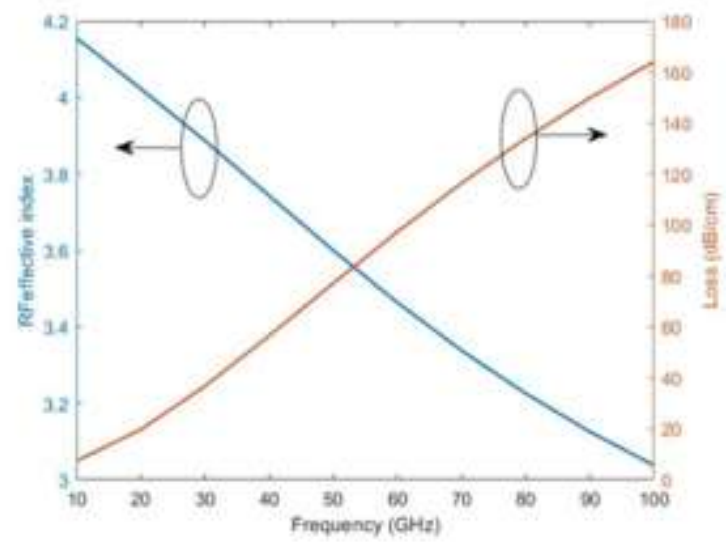

Fig 7: RF effective index and Loss acquired with respect to frequency

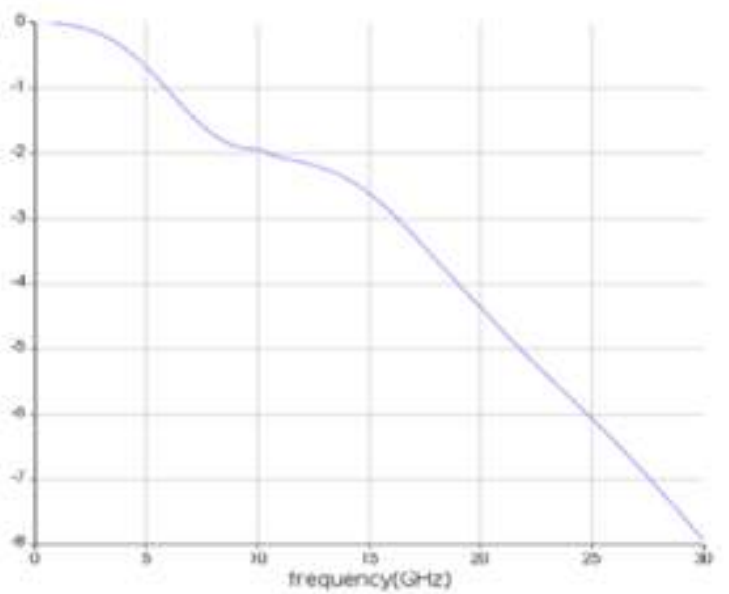

Fig 8: Electrical S21 (dB) with respect to frequency

\section{b. System-level analysis}

The dynamic performance of an unbalanced MZM incorporated with the designed phase shifter (PS-MZM) is studied. The PS-MZM has an intentional length variation of $100 \mu \mathrm{m}$ with longer arm provided a fixed bias voltage of $0.5 \mathrm{~V}$ and an alternating voltage swing $\left(\mathrm{V}_{\mathrm{pp}}\right)$ with DC reverse bias $\left(\mathrm{V}_{\mathrm{dc}}\right)$ is applied to the other arm. The pseudo-random bit sequence (PRBS) generator at $80 \mathrm{Gbps}$ produces the message data. An electrical signal generator with NRZ line coding technique was used to convert logical data into electrical message signal. The $\mathrm{CW}$ Laser generates the carrier signal at $1552.5 \mathrm{~nm}$. The received modulated signal is demodulated with the help of a photodetector with $1 \mathrm{~A} / \mathrm{W}$ responsivity. Bit error rate (BER) tester and eye diagram analyser was used to analyse the demodulated signal. An eye diagram with extinction ratio (ER) of $12.39 \mathrm{~dB}$ and BER of $8.67 \times 10^{-8}$ for a $\mathrm{V} \pi \mathrm{L}$ of $1.05 \mathrm{~V} . \mathrm{cm}$ at $3 \mathrm{~V}_{\mathrm{pp}}$ and $1.5 \mathrm{~V}_{\mathrm{dc}}$ is obtained. The eyecrossing at around $50 \%$ and wide eye opening leads to minimum duty cycle distortion and low inter-symbol interference. This ensures that PS-MZM is suitable for highspeed data rate applications. The energy per bit utilisation $\left(E_{b i t}=\frac{C V^{2}}{4}\right)$ for the data transmission is calculated to be $1.71 \mathrm{pJ} / \mathrm{bit}$. The performance of the PS-MZM is compared with the results of published articles with depletion type MZM in table 1 .

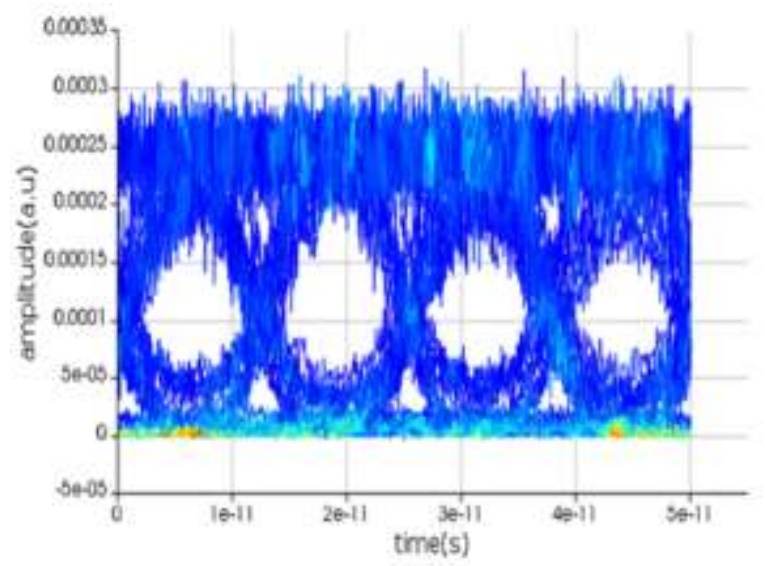

Fig 9: Eye diagram for PS-MZM with $V \pi L=1.05 \mathrm{~V} . \mathrm{cm}$

Table 1: Parameter Comparison With Published Results

\begin{tabular}{ccccc}
\hline Ref & $\begin{array}{c}\mathbf{L} \\
(\mathbf{m m})\end{array}$ & Gbps & $\begin{array}{c}\mathbf{V} \boldsymbol{\pi} \mathbf{L} \boldsymbol{\pi} \\
(\mathbf{V . c m})\end{array}$ & $\begin{array}{c}\text { ER } \\
(\mathbf{d B})\end{array}$ \\
\hline$[19]$ & 0.75 & 40 & 1.5 & 7.01 \\
{$[20]$} & 8 & - & 3.1 & 18 \\
{$[23]$} & 1.5 & 112 & 2.3 & - \\
{$[24]$} & 5 & 100 & 2.5 & 5.5 \\
{$[25]$} & 3 & 10 & 1.08 & 11 \\
{$[26]$} & 1.2 & 100 & 0.74 & 2.4 \\
This & 3.5 & 80 & 1.05 & 12.3 \\
work & & & & \\
\hline
\end{tabular}

Higher ER was obtained in [20] by increasing the length of the phase shifter. In [24], the phase shifter of length $5 \mathrm{~mm}$ is used to obtain the $\pi$ phase shift with ER of $5.5 \mathrm{~dB}$. The length was reduced to $1.2 \mathrm{~mm}$ [27] but reverse bias voltage of around $6 \mathrm{~V}$ is required, and $2.4 \mathrm{~dB}$ of ER was obtained. From table 1, it is clear that designed phase shifter in MZM performs better when compared with other published results.

Data centres span up to $15 \mathrm{~km}$ in length and are connected via fibre optic cables. The performance of PS-MZM for communications between chips connected via optic fibre cables is analysed. At $80 \mathrm{Gbps}$ and $\lambda=1552.5 \mathrm{~nm}$, the distance transmission capability is analysed. With the forward error correction (FEC) threshold of BER rate $1 \times 10^{-3}$ (between chips), it is observed from Fig. 9 that PS-MZM can transmit up to $29 \mathrm{~km}$. This ensures PS-MZM is suitable for intra data centre applications with guided wave transmission (optical fibre) and unguided wave transmission (free space optics). The transmission length can be improved with the help of an amplifier. 


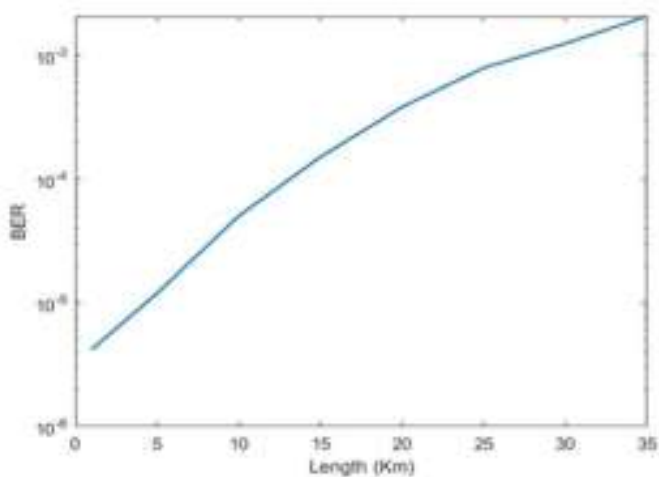

Fig 10: Transmission capability of PS-MZM in fibre optic cable at $80 \mathrm{Gbps}$

The speed capability analysis for PS-MZM is studied by varying the bit rate, and BER is calculated. With the increase in bit rate, BER increases as inferred from figure 10. It is observed that PS-MZM supports up to $95 \mathrm{Gbps}$. The bit rate support can be improved by increasing the PIN photo detector's responsivity or introducing a filter.

\section{Conclusion}

A plus-shaped PN junction phase shifter of $3.5 \mathrm{~mm}$ length is proposed to achieve high modulation efficiency. In the proposed design, the width of the $\mathrm{P}$ doped region in the rib is $100 \mathrm{~nm}$ and the thickness of $\mathrm{P}$ and $\mathrm{N}$ doped slab regions is $70 \mathrm{~nm}$. The designed phase shifter with silicon MZM at 80 Gbps, an ER of $12.39 \mathrm{~dB}$ and $8.67 \times 10^{-8}$ BER is obtained at $\mathrm{V} \pi \mathrm{L} \pi$ of $1.05 \mathrm{~V} . \mathrm{cm}$ for $1552.5 \mathrm{~nm}$ wavelength. The modulator is designed to meet the data centre futuristic demands and can also be used in other applications like optical switches, delay lines etc. The proposed phase shifter in MZM was also analysed for its supporting distance $(29 \mathrm{~km}$ for inter and intra data centre interconnect communication without an amplifier). Further improvement in bit rate can be obtained by the use of complex modulation formats.

\section{Reference}

[1] E. Perspectives and C. Report, "Cisco Annual Internet Report - Cisco Annual Internet Report (2018-2023) White Paper", Cisco, 2020. [Online]. Available: https://www.cisco.com/c/en/us/solutions/collateral/servi ce-provider/visual-networking-index-vni/white-paperc11-741490.html.

[2] H. Subbaraman et al., "Recent advances in siliconbased passive and active optical interconnects", Optics Express, vol. 23, no. 3, pp. 2487, 2015.

[3] D. Thomson et al., "Roadmap on silicon photonics", Journal of Optics, vol. 18, no. 7, pp. 073003, 2016.

[4] Q. Xu, S. Manipatruni, B. Schmidt, J. Shakya and M. Lipson, "125 Gbit/s carrier-injection-based silicon micro-ring silicon modulators", Optics Express, vol. 15, no. 2, pp. 430, 2007.

[5] W. Green, M. Rooks, L. Sekaric and Y. Vlasov, "Ultracompact, low RF power, $10 \mathrm{~Gb} / \mathrm{s}$ silicon Mach-Zehnder modulator", Optics Express, vol. 15, no. 25, pp. 17106, 2007.

[6] L. Chen, K. Preston, S. Manipatruni and M. Lipson, "Integrated $\mathrm{GHz}$ silicon photonic interconnect with micrometer-scale modulators and detectors", Optics Express, vol. 17, no. 17, pp. 15248, 2009.

[7] L. Alloatti et al., "100 GHz silicon-organic hybrid modulator", Light: Science \& Applications, vol. 3, no. 5, pp. e173-e173, 2014.

[8] N. Youngblood, Y. Anugrah, R. Ma, S. Koester and M. Li, "Multifunctional Graphene Optical Modulator and Photodetector Integrated on Silicon Waveguides", Nano Letters, vol. 14, no. 5, pp. 2741-2746, 2014.

[9] X. Zhang et al., "High Performance Optical Modulator Based on Electro-Optic Polymer Filled Silicon Slot Photonic Crystal Waveguide", Journal of Lightwave Technology, vol. 34, no. 12, pp. 2941-2951, 2016.

[10] J. Baek, J. You and K. Yu, "Free-carrier electrorefraction modulation based on a silicon slot waveguide with ITO", Optics Express, vol. 23, no. 12, pp. 15863, 2015.

[11] H. Dalir, Y. Xia, Y. Wang and X. Zhang, "Athermal Broadband Graphene Optical Modulator with $35 \mathrm{GHz}$ Speed", ACS Photonics, vol. 3, no. 9, pp. 1564-1568, 2016.

[12] S. Rajput, V. Kaushik, S. Jain and M. Kumar, "Slow Light Enhanced Phase Shifter Based on Low-Loss Silicon-ITO Hollow Waveguide", IEEE Photonics Journal, vol. 11, no. 1, pp. 1-8, 2019.

[13] S. Rajput, V. Kaushik, S. Jain, P. Tiwari, A. Srivastava and M. Kumar, "Optical Modulation in Hybrid Waveguide Based on Si-ITO Heterojunction", Journal of Lightwave Technology, vol. 38, no. 6, pp. 13651371, 2020.

[14] R. Soref and B. Bennett, "Electrooptical effects in silicon", IEEE Journal of Quantum Electronics, vol. 23, no. 1, pp. 123-129, 1987.

[15] A. Liu et al., "High-speed optical modulation based on carrier depletion in a silicon waveguide", Optics Express, vol. 15, no. 2, pp. 660, 2007.

[16] M. Ziebell et al., "40 Gbit/s low-loss silicon optical modulator based on a pipin diode", Optics Express, vol. 20, no. 10, pp. 10591, 2012.

[17] R. Jesuwanth Sugesh and A. Sivasubramanian, "Redesigning Mach-Zehnder Modulator with Ring Resonators", Lecture Notes in Electrical Engineering, pp. 185-191, 2017.

[18] P. Dong, L. Chen and Y. Chen, "High-speed lowvoltage single-drive push-pull silicon Mach-Zehnder modulators", Optics Express, vol. 20, no. 6, pp. 6163, 2012.

[19] H. Xu et al., "High speed silicon Mach-Zehnder modulator based on interleaved PN junctions", Optics Express, vol. 20, no. 14, pp. 15093, 2012.

[20] A. Rao et al., "High-performance and linear thin-film lithium niobate Mach-Zehnder modulators on silicon up to $50 \mathrm{GHz}$, Optics Letters, vol. 41, no. 24, pp. 5700, 2016.

[21] M. Félix Rosa et al., "Design of a carrier-depletion Mach-Zehnder modulator in $250 \mathrm{~nm}$ silicon-on- 
insulator technology", Advances in Radio Science, vol. 15, pp. 269-281, 2017.

[22] T. Ang, C. Png and S. Lim, "Numerical analysis and optimization of high-speed silicon microring resonator modulators using high-performance carrier-depletion phase shifters", Silicon Photonics XII, 2017.

[23] G. de Farias, Y. Bustamante, H. de Andrade, U. Moura, A. Freitas and D. de A. Motta, "Demonstration of > 48GHz Single-Drive Push-Pull Silicon Mach-Zehnder Modulator with Low $\mathrm{V}<\inf >\Pi</ \inf >\mathrm{L} ", 2018$ IEEE 15th International Conference on Group IV Photonics (GFP), Cancun, Mexico, 2018, pp 1-2.

[24] M. He et al., "High-performance hybrid silicon and lithium niobate Mach-Zehnder modulators for 100 Gbit s -1 and beyond", Nature Photonics, vol. 13, no. 5, pp. 359-364, 2019.

[25] K. Ogawa et al., "Silicon-based phase shifters for high figure of merit in optical modulation", Silicon Photonics XI, 2016.

[26] R. Palmer et al., "Silicon-Organic Hybrid MZI Modulator Generating OOK, BPSK and 8-ASK Signals for Up to $84 \mathrm{Gbit} / \mathrm{s} "$, IEEE Photonics Journal, vol. 5, no. 2, pp. 6600907-6600907, 2013.

[27] S. Jain, S. Rajput, V. Kaushik and M. Kumar, "High speed optical modulator based on silicon slotted-rib waveguide", Optics Communications, vol. 434, pp. 4953, 2019.

[28] S. Jain, S. Rajput, V. Kaushik, Sulabh and M. Kumar, "Efficient Optical Modulation With High Data-Rate in Silicon Based Laterally Split Vertical p-n Junction", IEEE Journal of Quantum Electronics, vol. 56, no. 2, pp. 1-7, 2020.

[29] T. Baehr-Jones et al., "Ultralow drive voltage silicon traveling-wave modulator", Optics Express, vol. 20, no. 11, pp. 12014, 2012.

[30] J. Dakin and R. Brown, "Handbook of Optoelectronics (Two-Volume Set)", CRC press, 2006.

[31] L. Binh, Optical multi-bound solitons, 1st ed. 2015.

\section{Creative Commons Attribution License 4.0 (Attribution 4.0 International, CC BY 4.0)}

This article is published under the terms of the Creative Commons Attribution License 4.0

https://creativecommons.org/licenses/by/4.0/deed.en US 